\title{
Identificación de asociaciones y complicidades, vía- email, de un grupo de analistas de redes sociales: ¿Qué intercambian los rederos?
}

\author{
Juan Felipe Nuñez \\ Ernesto Cárdenas \\ Departamento de Desarrollo Rural, Área de Evaluación y planeación de proyectos \\ de desarrollo rural en zonas marginadas Colegio de Postgraduados, México ${ }^{1}$
}

\begin{abstract}
Resumen
Las comunidades de investigadores en el mundo son de los pocos conglomerados que están permanentemente abiertos, en y con su entorno; por lo tanto ofrecen información valiosa sobre su génesis, desarrollo y prospectiva, y tal vez indicios de posibles paradigmas que estén, en forma embrionaria, generándose en el interior de dichos colectivos. Si bien el ARS es un enfoque que se ha venido desarrollando poco a poco durante los últimos 50 años, en la actualidad se puede observar, alrededor de este tema, una comunidad de investigadores en ciernes, buscando su propio proceso de maduración en las ciencias sociales. Una de las vías de búsqueda de este grupo es la construcción reticular dada a través del intercambio de insumos de conocimiento que se ha generado entre sus miembros por medio de diversos canales, por ejemplo, el uso de las llamadas TIC's. En esta dirección, conviene llamar la atención sobre este tipo de comunidades nuevas que intentan construir una plataforma de conocimiento al interior de una de las disciplinas que se ha caracterizado, por norma y obligatoriedad, deconstruir continuamente el trabajo epistemológico.
\end{abstract}

Palabras clave: Comunidad, grupo, red, investigación.

\begin{abstract}
Research communities in the world are among the few social conglomerates that are permanently open with their surroundings, therefore they offer valuable information about its origins, development and future, and probably evidence about possible scientific paradigms that are in embryonic form in such groups.

Although NSA is an approach that has been developed over the last 50 years, actually is possible see, around this topic, a budding research community which is looking its own maturation process in the social sciences.

One way to search for this group is with the lattice construction through exchange of knowledge's inputs that were generated among its members with the help of different social channels and the use of information and communication technologies. In this direction, it should draw attention to these new communities trying to build a platform of knowledge within a discipline that has been characterized as a deconstructor of the epistemological work.
\end{abstract}

Keywords: Community, network, research group.

\footnotetext{
1 Dirección de contacto: Juan Felipe Nuñez: nunezej@colpos.mx.
} 
"Pensamos en generalidades, pero vivimos en detalles"

(Alfred North Whitehead)

\section{Introducción}

Desde que Mark Granovetter investigó, en la década de los 70, el proceso de difusión de información entre personas para conseguir empleo, por medio de las redes sociales que estas establecían (Scott, 1996; Granovetter, 1973), e integró diversas tradiciones metodológicas que daban cuenta, desde sus propias posiciones, de este proceso de construcción social, dio inicio a lo que posteriormente ha sido llamado Análisis de Redes sociales (ARS), un campo que comenzó a crecer en sus aplicaciones y pretensiones epistemológicas al conjugar, de forma más o menos afortunada -hasta ahora-, dichas posiciones en sus explicaciones de la reticularidad del mundo de lo real. Desde ese momento, hasta ahora, el ARS o Social Network Analysis (SNA) es un campo de investigación de las ciencias sociales que sigue integrando diversas herramientas, conceptos y/o nociones metodológicas provenientes de otros campos y/o disciplinas (criminalística, matemática de grafos, TIC's, psicología, lingüística, etc.). Con esto, se logra recuperar e innovar un lenguaje, en y para el análisis de lo social, con el fin de ofrecer nuevas explicaciones sobre las dinámicas de agrupación de individuos y comunidades humanas a fin de encontrar $y$ analizar patrones $\mathrm{y} / \mathrm{o}$ tendencias a nivel macrosocial que no eran visibles bajo las ópticas tradicionales de las ciencias sociales.

Algunas de las tradiciones de las ciencias sociales que han confluido en el uso del ARS son: el concepto antropológico de la estructura social como un entramado reticular entre los individuos; la concepción sociológica de la estructura social como "red social" que afecta a la sociedad como un todo; y las explicaciones "estructurales" del proceso comunicacional como ente político, social, cultural y científico, diferenciado, en el mundo (Castells, 2001). Estas vertientes (entre otras) innegablemente revelan la raíz estructuralista de este enfoque ya que permite la descripción, clasificación, análisis e intervención de las estructuras de intercambio entre los sujetos sociales, así como las posibilidades y limitantes para el desarrollo de objetivos organizacionales agrupados en acciones colectivas o individuales (Vélez, 2007).

En esta dirección, a través de la cuantificación de lo cualitativo, el ARS ha desarrollado un campo que le permite ser considerado como instrumento de medición y 
visualización de la dimensionalidad de las estructuras sociales que surgen de las relaciones establecidas entre múltiples actores sociales (comunidades, organizaciones, naciones, etc.) así como de la redundancia y reproducción de dichas conexiones (White et al. 2000). Esto es, puede proveer un análisis con carácter matemático y visual de sistemas humanos complejos, así como de las interacciones establecidas entre los componentes de dichos sistemas (personas, grupos, organizaciones u otras entidades procesadoras de información y/o conocimiento) (Navarro y Salazar, 2007), de forma ilustrativa y breve (Velázquez y Aguilar, 2005). Además de lo anterior, el ARS se ha popularizado ya ha logrado abordar relaciones estructurales que otros métodos ignoraban (Schmidt, 2009) o estaban imposibilitados para analizar.

\section{ARS y ciencias sociales}

EI ARS ha demostrado tener un crecimiento dinámico dentro de las ciencias sociales y, hasta el momento, se ha aplicado en temas tan diversos como salud, psicología, organización empresarial y comunicación electrónica, desarrollo rural (Clark, 2006), etc. Ha aportado puntos de vista distintos sobre temas de sociología de las organizaciones, etnias y contactos sexuales. Actualmente, el ARS ha incurrido en la investigación sobre la Web, como macro estructura de agrupación social y mecanismo con el potencial para permitir la generación de ciencia (Monsalve, 2008). Algunos otros campos o temáticas de análisis en los que las redes han mostrado su aplicación son: clases escolares y escuelas, despachos, bandas o grupos, clubes sociales, laboratorios u otros lugares de trabajo. También se han realizado estudios de organizaciones de más envergadura: élites políticas (Gil, 2005), redes en la economía nacional, redes de científicos en una disciplina profesional y relaciones internacionales. Dentro del marco organizacional $\mathrm{y} / \mathrm{o}$ institucional el ARS ha abordado relaciones de poder $\mathrm{y}$ autoridad en

organizaciones complejas formales como son empresas, consejos de administración, clases políticas. Las relaciones de afinidad y de amistad han sido también una materia habitual de las redes sociales; así como las de familia y parentesco, principalmente en temas como roles sociales, familias o matrimonios. Otra temática corriente se refiere a las relaciones de comunicación: lazos entre actores como canales de transmisión de mensajes; de transacción: actores que intercambian instrumentos físicos o simbólicos, regalos, venta económica, compras, servicios, información sobre trabajo, aborto, consejos políticos, trabajo social (Lozares, 1996). Con esto, se puede decir que el ARS 
propone toda una topología de la acción social, de la praxis, al interior de las ciencias sociales.

\section{ARS, alcances y/o limitaciones}

En relación con lo anterior, proponemos que las unidades de análisis del ARS sean dos. En primer lugar, están las relaciones establecidas entre los sujetos; por lo que su atención se centra, primordialmente, en las condicionantes estructurales de las acciones de las personas (Sanz, 2003), es decir, las particularidades que poseen estas relaciones. En segundo lugar, dado el carácter nominativo de los vínculos, se genera un producto inevitable de dichas particularidades, es decir, el sujeto social.

En esta dirección, consideramos que el concepto de Individuo se vuelve inoperante y contradictorio en y para el propio ARS ya que al estudiar relaciones más que los atributos de los individuos, el concepto de Sujeto es más preciso debido a que designa el atributo de la sujeción, de lo relacionado, de la unidad dividida por sus relaciones, en cambio Individuo señala la cualidad de lo indivisible. En este sentido se propone trabajar, principalmente, con la categoría Sujeto, pero sin dejar de reconocer que cada Sujeto es una individualidad encarnada de decisiones.

Lo anterior permite al ARS: a) identificar la relación existente entre los tipos de redes sociales y los recursos que fluyen a través de ella; por lo tanto b) intervenir eficazmente en las estructuras reticulares y modificar el flujo y acceso a los recursos que los miembros de una red pudieran tener.

En este sentido, el ARS es usado para generar mapas de relaciones a fin de mejorar el análisis y la eficiencia de la estructura organizacional de las empresas, desarrollo de nuevos productos, análisis de transmisión de enfermedades, propagación de virus informáticos, análisis del comportamiento de comunidades, estudio del capital social, entre otros temas.

Por otra parte, la integración, más o menos exitosa, de diversas herramientas metodológicas, la incorporación de un lenguaje diferente al análisis de lo social, la expresión de los resultados a través de un vocabulario técnico que concilia la noción teórica y la aplicación y visualización del dato procesado a través de programas software de análisis de redes sociales, así como el estudio de otros eventos sociales 
que otros métodos ignoraban o estaban imposibilitados de sistematizar, hacen del ARS un enfoque novedoso y poseedor de un valor adicional que cualquier científico social puede aprovechar; sin embargo, las investigaciones que hacen uso del ARS, no deben de ser consideradas irrefutables y/o infalibles ya que están sujetas a las mismas necesidades de validación y a la presencia de los errores que afectan a cualquier investigación de lo social. En consonancia con esto, los trabajos basados en el ARS están sujetos, en principio, a las mismas restricciones de fidelidad y consistencia de los datos que cualquier otra investigación está obligada a tener, por lo que se vuelve necesario reconocer, como atípica, aquella situación en la que la información disponible y/o registrada se encuentra predispuesta para dar cuenta de la lógica reticular (Miceli, 2008). Dicho condicionamiento señala directamente al ángulo subjetivo del investigador de lo social. De hecho, la incorporación de elementos, como los señalados al principio de este párrafo, hace del ARS un objeto de estudio que es necesario analizar y monitorear constantemente.

En este contexto, este enfoque llama la atención por dos matices. Además de su capacidad para integrar otras herramientas metodológicas y/o que permite analizar la integración social, él mismo es un campo de discusión y construcción social ergo es un crisol integrador donde se dan cita, sujetos y colectivos sociales, para desarrollar la epistemología de esta herramienta de análisis. En este esfuerzo se logra observar la construcción y movimiento de una comunidad especializada en el tema de redes sociales.

Esta característica ha permitido la construcción de grupos multidisciplinarios, así como la apertura de un amplio abanico de temas para estudiar y/o intercambiar. Los analistas de las redes sociales no son un grupo aislado de las reglas de agrupamiento que analizan. Ellos mismos son sujetos sociales que buscan agruparse de múltiples formas con su entorno, tal y como se observa en el presente documento.

\section{Limitaciones}

Este es un estudio que se limita a los intercambios de e-mails en una comunidad dada. De hecho, de acuerdo a la amplitud de la información, no se exploró y/o analizó a profundidad el carácter y/o tipo de los artículos intercambiados y/o recomendados (por tema, por autor, por tendencia, etc.) a través del LISTSERV, así como el material 
contenido en los diversos sitios webs sugeridos, tampoco se analizó el tipo y/o contenido de los cursos, conferencias y/o congresos a los que se invitó a los adscritos a este sitio. Esto hubiera permitido agrupar a los rederos, probablemente, en escuelas

de trabajo y/o conceptuales al interior del ARS y generado un análisis más sólido sobre la dinámica epistemológica de este enfoque en este grupo de analistas de redes sociales, así como un análisis reticular del discurso construido alrededor del propio ARS.

De igual forma, faltó llevar a cabo un análisis sobre el papel del propio LISTSERV en la conformación de toda una tipología de redes sociales, así como comparar las relaciones cara a cara o colaboración fuera de la LISTSERV con las generadas en este último.

Es importante notar que no se analiza la complejidad involucrada en la conformación de esta comunidad, ya que es un trabajo que implica el uso de una cantidad de recursos que no se poseen en este momento. Por lo tanto, este trabajo se circunscribe solamente a la identificación de los temas intercambiados por medio de e-mail y las agrupaciones resultantes de dicho intercambio. En relación con esto, el presente documento no pretende más que dar un acercamiento general a las dinámicas de agrupamiento de una comunidad de especialista en ARS.

\section{Método}

Para llevar a cabo este trabajo, se planteó la siguiente pregunta:

¿Cuáles son los insumos y/o recursos de información que se intercambian al interior de un grupo de analistas de redes sociales, agrupados en una determinada estructura reticular?

Para responder a dicha pregunta se resolvió agrupar, clasificar y analizar el conjunto de correos electrónicos enviados y/o compartidos, entre el año 2009 y 2012, a través del correo de la Revista Hispana para el Análisis de Redes Sociales: "REDES@LISTSERV.REDIRIS.ES" y que Ilegaron a la bandeja de entrada del correo nunezej@catunesco.upc.edu, que pertenece a uno de los autores del presente documento. Los correos se agruparon por fecha de envío (día, mes y año). Los temas de los correos fueron agrupados en 59 áreas de intercambio. Esto permitió utilizar las herramientas del ARS y ver, por medio del programa Visone, agrupamientos alrededor 
de áreas de intercambio, así como la representación de las estructuras reticulares conformadas en torno a los temas intercambiados.

\section{Resultados}

Se analizaron 1,456 correos generados por un grupo de 174 remitentes y 79 destinatarios. El destinatario que permitió reunir toda esta información, fue REDES@LISTSERV.REDIRIS.ES, que agrupó el 80\% de los e-mails, aunque este actor también permitió espacios de comunicación e intercambio alternos entre este grupo de rederos. El $N^{\circ}$ de temas, relacionados con el ARS, que se intercambiaron en dicha comunidad, fue de 59 tópicos generales (Tabla 1).

Tabla 1. Tipo de temas del ARS que fueron intercambiados (2009-2012).

\begin{tabular}{|c|c|c|}
\hline Amistad & Economía & Metodología \\
\hline Antropología & Educación & Migración \\
\hline Arqueología & Ego/negocio & Muestreo \\
\hline Arte & Entrevista & Organización \\
\hline Becas & Etnicidad & Otros \\
\hline Bibliografía & Futbol & Pobreza \\
\hline Biología & Geodésico & Política \\
\hline Capital social & Herramientas & Preguntas \\
\hline Centralidad & Humor & Prensa \\
\hline Ciencia & Infantes & Psicología \\
\hline Cliques & Intercambios & Recomendación de webs/links \\
\hline Conferencias/congresos & Lazos/argumentación & Riesgo \\
\hline Consulta generales & Libros/artículos & Salud \\
\hline
\end{tabular}




\begin{tabular}{|l|l|l|}
\hline Cooperación & Lingüística & Saludos/agradecimientos/felicitaciones \\
\hline Cuestionario & Manual & Sienna \\
\hline Cursos/academia & Mapa & Socializar \\
\hline Definiciones & Marxismo & Software/programas \\
\hline Tesis & Trabajo & Ucinet \\
\hline TICS & Twitter & Violencia \\
\hline Desarrollo rural & Matrices & \\
\hline
\end{tabular}

Esta diversidad de temas se amplificó conforme fue avanzando dicho intercambio (Gráfico 1). En el 2009, primer año registrado en la muestra, se realizó el $13.32 \%$ del total de intercambios; entre el 2010 y el 2011, hubo un ritmo desigual en estos movimientos; sin embargo, para el 2012, último año de registro, el $n^{\circ}$ de intercambios entre los usuarios fue del $38.46 \%$ señalando un crecimiento exponencial en los intercambios de la comunidad de rederos, por lo tanto en sus dinámicas de cohesión.

Gráfico 1. Evolución en el $n^{\circ}$ de temas intercambiados, a través de e-mail, en una población especializada en el ARS.

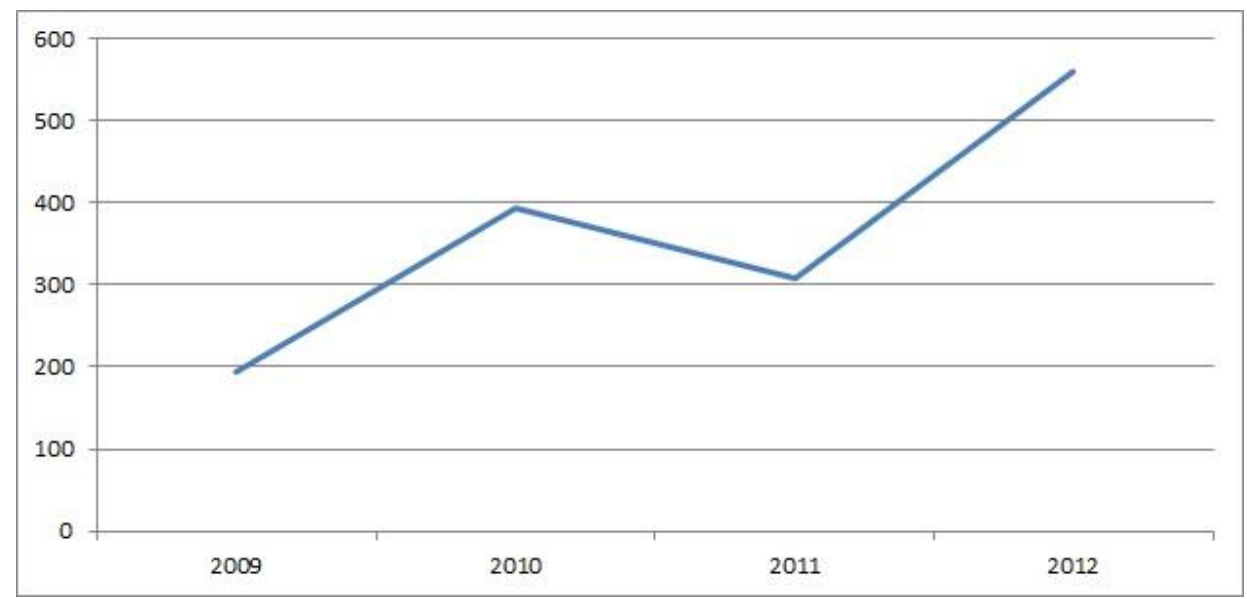

La mayor parte de los temas intercambiados (64.70\%) por este grupo de rederos se concentró (Tabla 2) en los rubros sobre sitios webs, foros de intercambio, bibliografía 
especializada, renovación y/o establecimiento de lazos sociales, dudas puntuales sobre el ARS y socialización de diversos tipos de información sobre ARS. Todos estos elementos señalan la presencia de una comunidad incipiente pero dinámica en sus intercambios, aún a pesar de la distancia geográfica que pudiera existir entre los miembros.

Tabla 2. Temas de intercambio, a través de e-mail, entre un población especializada en el ARS. 2009-2012.

\begin{tabular}{|l|c|c|}
\hline \multicolumn{1}{|c|}{ Atributos } & Frec & \% \\
\hline Recomendación de webs/links de ARS & 197 & 13.53 \\
\hline Conferencias/congresos de ARS & 192 & 13.19 \\
\hline Libros/artículos sobre ARS & 187 & 12.84 \\
\hline Saludos/agradecimientos/felicitaciones & 141 & 9.68 \\
\hline Consulta sobre ARS & 117 & 8.04 \\
\hline Socializar información diversa & 108 & 7.42 \\
\hline
\end{tabular}

Estas dinámicas de agrupación, entre los colectivos que han impulsado la lista REDES@LISTSERV.REDIRIS.ES, se logran observar, con mucho más detalle, cuando se hace la descripción por cada uno de los cuatro años analizados (2009-2012). Las temáticas intercambiadas indican las preocupaciones de esta comunidad. En relación con esto, en el año 2009, los temas principales fueron recomendaciones de sitios webs; renovación y/o establecimiento de lazos sociales e intercambio de títulos de libros y/o artículos, entre otros. (Tabla 3)

Tabla 3. Temas de intercambio, a través de e-mail, entre una población especializada en el ARS. Año 2009.

\begin{tabular}{|l|l|l|}
\hline \multicolumn{1}{|c|}{ Conceptos } & \multicolumn{1}{|c|}{ Frec } & $\%$ \\
\hline Recomendación de webs/links & 53 & 27.32 \\
\hline Saludos/agradecimientos/felicitaciones & 14 & 7.22 \\
\hline Libros/artículos & 12 & 6.19 \\
\hline Manual & 10 & 5.15 \\
\hline Ciencia & 8 & 4.12 \\
\hline Conferencias/congresos & 8 & 4.12 \\
\hline
\end{tabular}

En el año 2010, (Tabla 4), al tema de las recomendaciones de sitios webs se añadieron, como principales tópicos, los temas de bibliografía, foros de intercambio, 
uso de la plataforma twitter para analizar y/o establecer redes sociales, socialización de diversos temas sobre el ARS, así como el uso del ARS para analizar el futbol.

Tabla 4. Temas de intercambio, a través de e-mail, entre una población especializada en el ARS. Año 2010.

\begin{tabular}{|l|l|l|}
\hline \multicolumn{1}{|c|}{ Conceptos } & Frec & \% \\
\hline Recomendación de webs/links & 54 & 13.71 \\
\hline Libros/artículos & 52 & 13.20 \\
\hline Conferencias/congresos & 40 & 10.15 \\
\hline Twitter & 39 & 9.90 \\
\hline Socializar & 27 & 6.85 \\
\hline Futbol/ & 24 & 6.09 \\
\hline
\end{tabular}

Para el año 2011 (Tabla 5) el tema de recomendación de sitios web, sigue siendo importante, sin embargo, el principal tema que se intercambió en este año fue sobre foros de intercambio como congresos y conferencias. En tercer lugar se ubica la socialización de diversos temas sobre el ARS, le sigue la renovación y/o establecimiento de lazos sociales, así como el intercambio de bibliografía.

Tabla 5. Temas de intercambio, a través de e-mail, entre una población especializada en el ARS. Año 2011.

\begin{tabular}{|l|l|l|}
\hline \multicolumn{1}{|c|}{ Conceptos } & \multicolumn{1}{c|}{ Frec } & \multicolumn{1}{c|}{$\%$} \\
\hline Conferencias/congresos & 67 & 21.75 \\
\hline Recomendación de webs/links & 33 & 10.71 \\
\hline Socializar & 33 & 10.71 \\
\hline Saludos/agradecimientos/felicitaciones & 32 & 10.39 \\
\hline Libros/artículos & 26 & 8.44 \\
\hline Consulta sobre Ars & 16 & 5.19 \\
\hline
\end{tabular}

Para el año 2012, (Tabla 6), las dinámicas de intercambio, se concentraron alrededor de la construcción e intercambio de fuentes de documentación sobre el ARS, consulta sobre diversos parámetros del enfoque de redes, la participación en foros de intercambio, así como la renovación y/o fortalecimiento de lazos sociales, entre otros. 
Tabla 6. Temas de intercambio, a través de e-mail, entre una población especializada en el ARS 2012

\begin{tabular}{|l|l|l|}
\hline \multicolumn{1}{|c|}{ Conceptos } & Frec & $\%$ \\
\hline Libros/artículos & 97 & 17.32 \\
\hline Consulta & 81 & 14.46 \\
\hline Conferencias/congresos & 77 & 13.75 \\
\hline Saludos/agradecimientos/felicitaciones & 75 & 13.39 \\
\hline Recomendación de webs/links & 57 & 10.18 \\
\hline Socializar & 43 & 7.68 \\
\hline
\end{tabular}

Este continuo intercambio de diferentes tópicos, a lo largo de cuatro años, a través del REDES@LISTSERV.REDIRIS.ES, permite verificar un movimiento intermitente de agrupación de la comunidad analizada, así como el movimiento de las preocupaciones, de esta comunidad alrededor del ARS. Por ejemplo, el tema sobre plataformas de internet, como el vehículo más funcional e impersonal para intercambiar información, aparece como primera opción en el 2009 y 2010; para el 2011, este tema es segundo en importancia y, para el 2012, se vuelve un tema secundario (cae al quinto lugar).

El tema Saludos, como una forma para establecer y/o reformular lazos sociales al interior de dicha comunidad, aparece en segundo lugar en el 2009; en el 2010 no se registra dentro de los seis principales temas de intercambio, aunque en el 2011 y 2012, es el cuarto tema intercambiado por la población analizada.

Otro tema que resulta de interés es sobre la recomendación de Libros/artículos. Este tema es el tercer tipo de insumo intercambiado en el 2009, y el segundo en 2010, aunque en 2011 solo es el quinto tema en importancia; sin embargo, en 2012, es el principal tema intercambiado en este grupo. Este tópico, probablemente se refiera a la ampliación de la base epistemológica del ARS, ya que dichos materiales comienzan a ser revisados, analizados y/o comentados por los analistas del tema.

En el 2009, el tema de Conferencias/congresos es el sexto material en importancia que intercambia el colectivo de rederos. En 2010, dicho tema es el cuarto en importancia. En 2011, este tema es la principal preocupación de los analistas del ARS. Para 2012, el tema se degrada al tercer lugar del trueque reticular. Cabe señalar que este insumo puede estar indicando la preocupación y necesidad de comenzar a conocer e 
intercambiar información "face to face" con otros rederos para tener un acercamiento más sólido y definir aún más el insumo de confianza entre los colectivos del ARS.

La evolución del intercambio, en los temas señalado, permite observar dinámicas de agrupación y cohesión de la comunidad alrededor de intereses de investigación diversos y particulares. Al mismo tiempo, detrás de cada interés se observa el objetivo de probar la solidez de la herramienta ante múltiples fenómenos. Esto indica la necesidad de una comunidad de construir una determinada confianza sobre el ARS como instrumento de análisis del mundo de lo social y como vehículo de vinculación. Además de esto, la evolución en la diversidad de temas en las que se hace intervenir al propio ARS señala un determinado grado de ductilidad epistemológica del enfoque, como se muestra en los principales temas intercambiados por la comunidad (Gráfico 2).

Por otro lado, si se parte de que el actor REDES@LISTSERV.REDIRIS.ES abrió procesos de intercambio entre otros actores, se puede aventurar la idea de que también fue impulsor de comunidades alternas. En este sentido se pueden analizar, incluso, aquellos datos reticulares de estas comunidades independientes sin tomar en cuenta el peso de dicho actor (aunque integre el $80 \%$ de la información recibida). Esto permitió analizar, en términos generales, un total de 290 e-mails que fueron enviados entre 2009 y 2012, sin considerar aquellos correos recibidos por REDES@LISTSERV.REDIRIS.ES (Tabla 7). ${ }^{2}$ Los temas intercambiados en estas comunidades al margen, fueron 32 (Tabla 7).

\footnotetext{
2 De aquí en adelante, los cuadros y análisis presentados en este documento serán hechos con referencia solamente a los datos intercambiados directamente entre los miembros de la comunidad de rederos, sin considerar los datos que fueron enviados directamente a REDES@LISTSERV.REDIRIS.ES, a excepción de aquellos donde se indique lo contrario.
} 
Tabla 7. Tipo de temas del ARS que fueron intercambiados entre una población especializada en el ARS (2009-2012).

\begin{tabular}{|l|l|l|}
\hline Antropología & Futbol & Política \\
\hline Ciencia & Herramientas & Preguntas \\
\hline Cliques & Lazos/argumentación & Prensa \\
\hline Conferencias/congresos & Libros/artículos & Recomendación de webs/links \\
\hline Consulta & Manual & Salud \\
\hline Cooperación & Matrices & Saludos/agradecimientos/felicitac \\
\hline Cuestionario & Metodología & Sienna \\
\hline Cursos/academia & Migración & Socializar noticias \\
\hline Desarrollo rural & Muestreo & Software/programas \\
\hline Educación & Pobreza & Tesis \\
\hline Twitter & Ucinet & \\
\hline
\end{tabular}

El 74\% de estos temas (Tabla 8 ) se concentró en 4 tópicos: Saludos, recomendación de plataformas webs, recomendación de libros y/o artículos, así como socialización de diversas noticias relacionadas con el ARS. 
Gráfico 2. Temas de ARS intercambiados por e-mail, por año, entre un población de especialistas en ARS. 2009-2012.

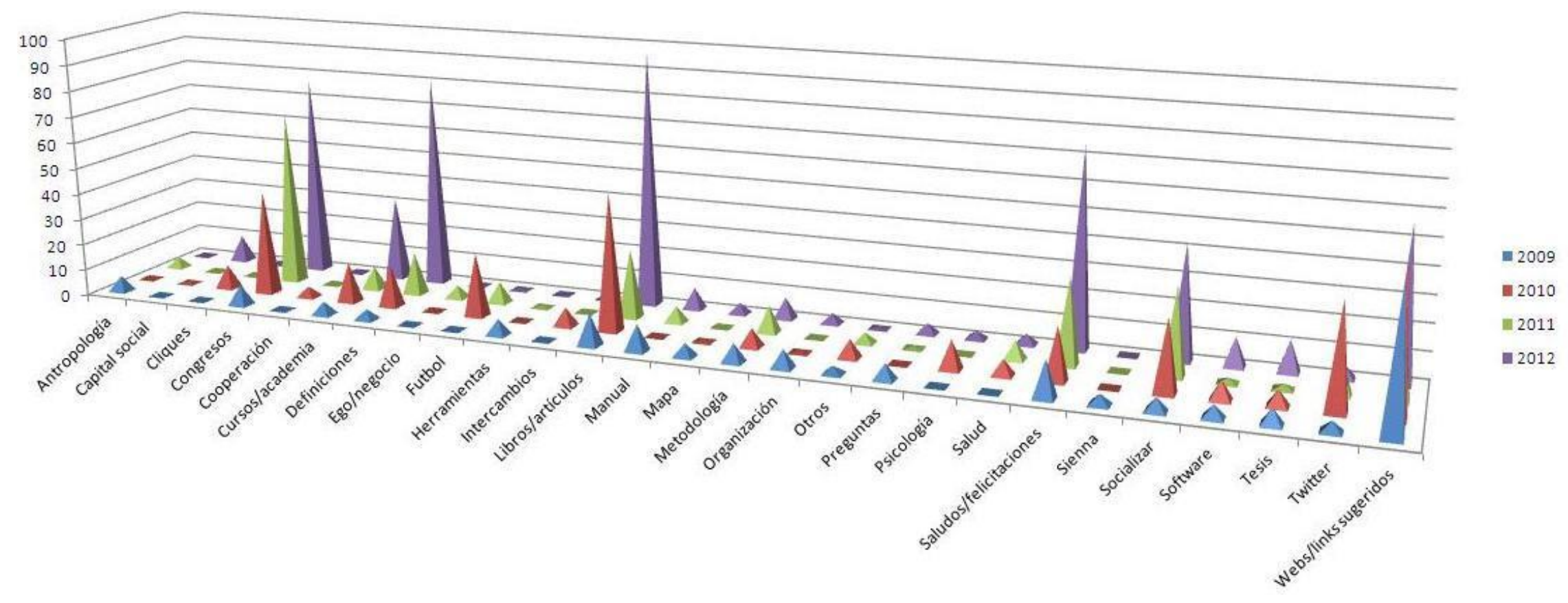


Tabla 8. Principales temas de intercambio, a través de e-mail, entre una comunidad de rederos. 20092012.

\begin{tabular}{|l|l|l|}
\hline \multicolumn{1}{|c|}{ Conceptos } & \multicolumn{1}{c|}{ Frec } & \\
\hline Saludos/agradecimientos/felicitaciones & 98 & 33.79 \\
\hline Recomendación de webs/links & 48 & 16.55 \\
\hline Libros/artículos & 39 & 13.45 \\
\hline Socializar diversas noticias & 32 & 11.03 \\
\hline
\end{tabular}

Al hacer la revisión del tipo de materiales que esta comunidad intercambió entre sí, en cada uno de los años revisados, se observó que esta reciprocidad no varío mucho de la que se presentó en el cuadro anterior siendo los temas de Saludos, agradecimientos y/o felicitaciones, así como la Recomendación de plataformas webs los elementos de mayor agrupación de este colectivo.

Con lo anterior se ha dado un breve vistazo sobre la constitución de una comunidad especializada y los insumos que se intercambian al interior de esta red social. En relación con esto, la construcción de este colectivo lleva a plantear las siguientes cuestiones: ¿Quiénes constituyen este espacio reticular? ¿Cómo se dinamiza? ¿Quiénes son los actores que permiten dicha agrupación?

La utilización de software permite integrar y agrupar cantidades ingentes de información relacional y facilita la evaluación de estructuras sociales complejas. Con ayuda del programa Visone 2.6.5.(VDT, 2012), se lograron generar grafos que permitieron distinguir, en la estructura en red analizada, cúmulos sociales definidos alrededor de determinados actores (Gráfico 3), por lo tanto, grupos interrelacionados en torno a determinadas temáticas del ARS. En esta dirección, el Gráfico 3 representa, de forma general: a) Dinámicas de agrupamiento, b) Grupos de trabajo, c) Complicidades sociales, d) Liderazgo de determinados actores sobre el resto del colectivo. 
Grafico 3. Cúmulos sociales generados por los intercambios, vía e-mail, entre un población especializada en el ARS. 2009-2012. Programa Visone.

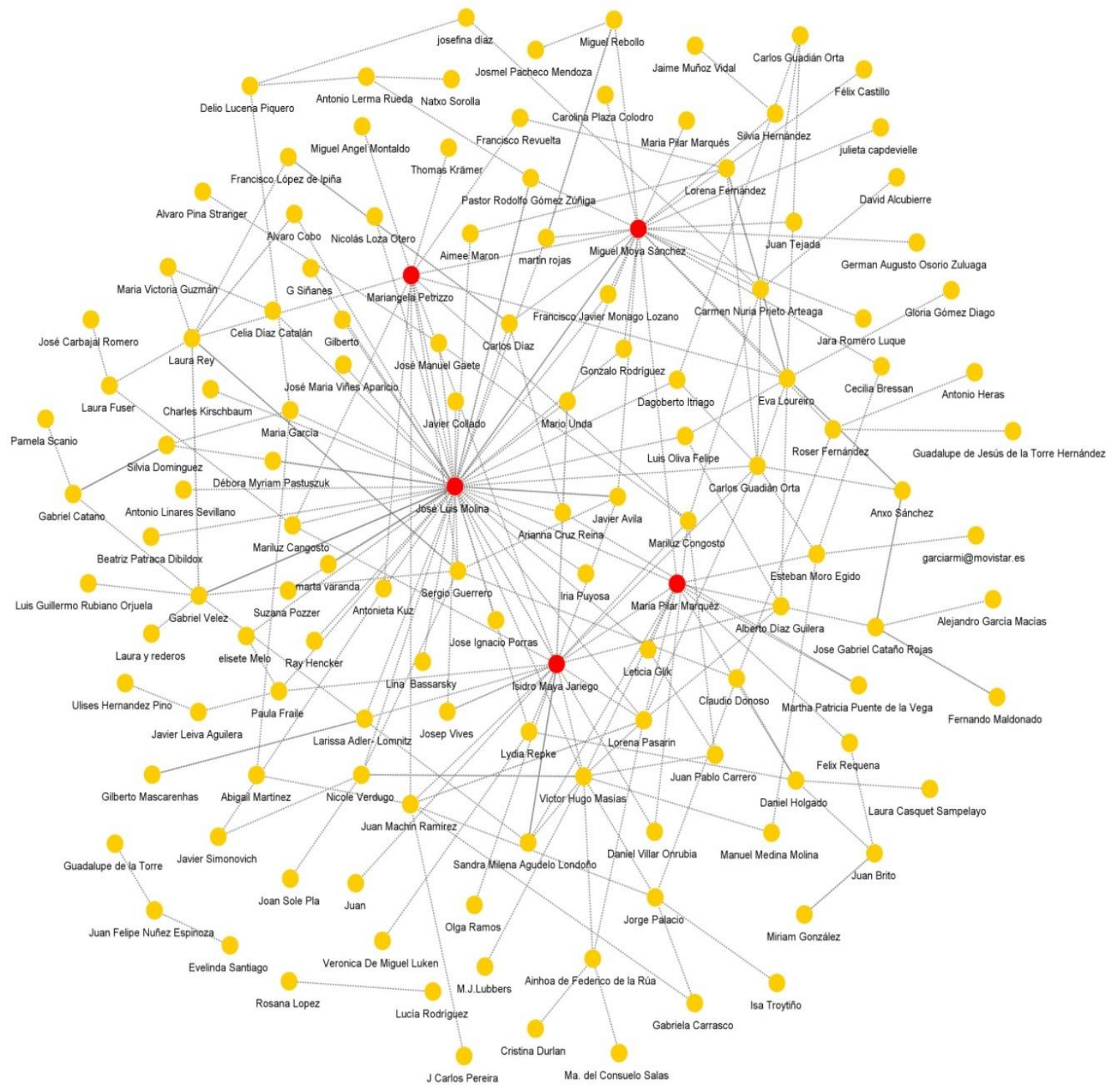

Al momento de revisar la densidad social de esta red, se observa un bajo desempeño ( $1.7 \%$ de todas las relaciones posibles están presentes). Esta densidad se refiere a procesos de centralización de la información (tal vez porque el medio analizado -e-mail- es un medio focalizado en un sitio que es gestionado por un determinado grupo de analistas del ARS). Aunque esto lleva a generar la siguiente cuestión clas redes sociales, vía e-mail, son poco densas debido a que no existe el "face to face" generado en las relaciones directas? 
El mapa relacional permite tener un detalle más valioso en cuanto a la identificación de actores centrales se refiere y la solidez de las relaciones establecidas entre ellos, vía e-mail. De esta forma, en la trama analizada (Gráfico 4) se observan, a través del grosor de los vínculos, grupos de trabajo "más íntimos", con más complicidades, en términos de la recurrencia de sus comunicaciones y/o temas, así como aquellos actores/equipo que tienen un peso determinante en dinamizar, a través de la correspondencia electrónica, la comunicación al interior de la estructura comunitaria analizada. De igual forma, esto permite visualizar al grupo nodal que está definiendo las principales aristas del paradigma en construcción.

Gráfico 4. Actores y vínculos centrales generados por los intercambios, vía e-mail, entre un población especializada en el ARS. 2009-2012. Programa Visone.

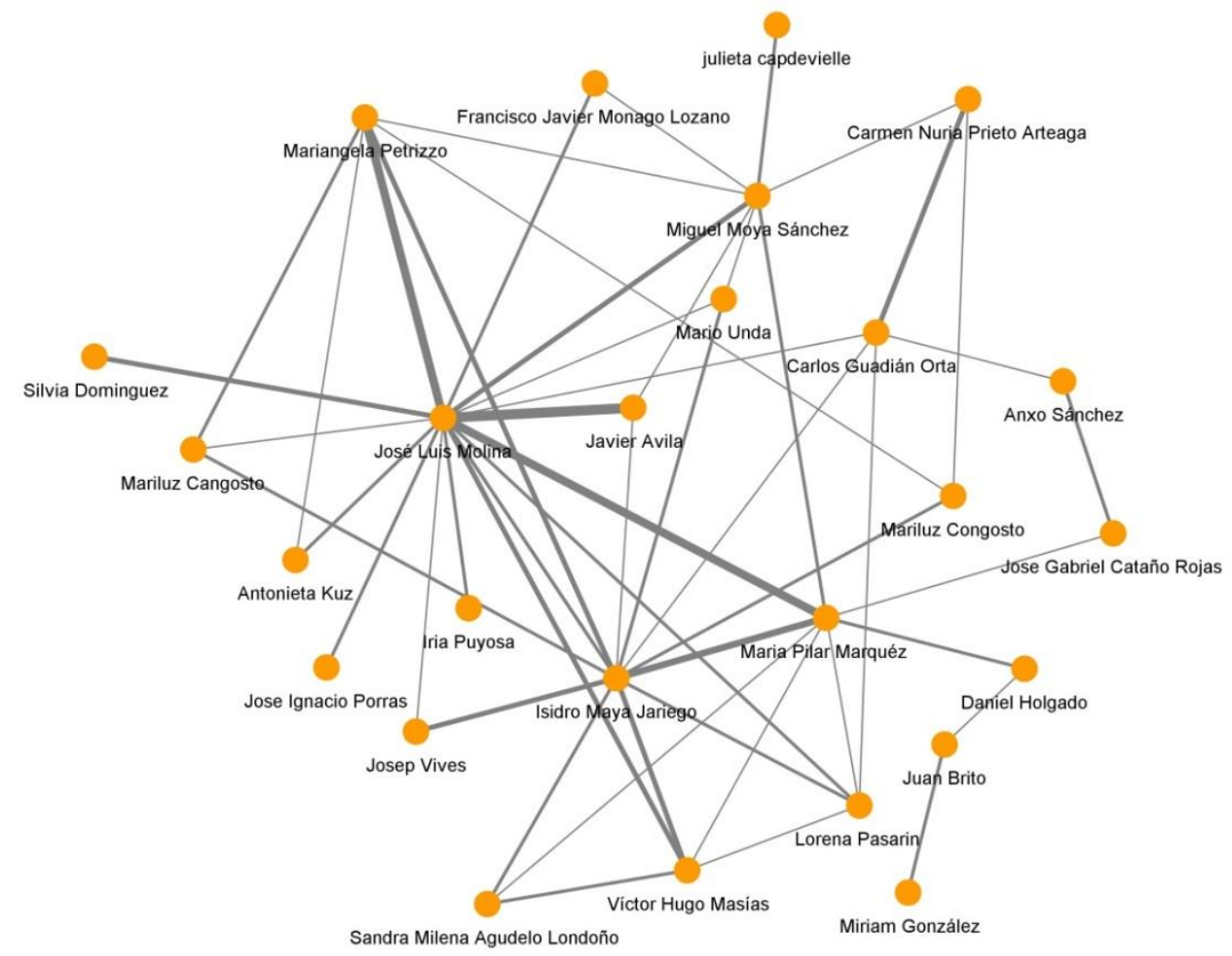

Los grupos de colaboración, mostrados en el grafo anterior, se logran observar con mucho mayor detalle al identificar equipos compactos de trabajo a través del concepto de Cliqués (Tabla 9). Con esta herramienta se identifica el flujo del insumo Afinidad a lo largo de dichos grupos. 
Tabla 9. Cliqués de 2 generados por los intercambios, vía e-mail, entre un población especializada en el ARS. 2009-2012. Programa UCINET.

\begin{tabular}{|l|l|}
\hline Gabriel Vélez & José Luis Molina \\
\hline Isidro Maya Jariego & José Luis Molina \\
\hline Javier Avila & José Luis Molina \\
\hline José Ignacio Porras & José Luis Molina \\
\hline José Luis Molina & Miguel Moya Sánchez \\
\hline José Luis Molina & Pastor Rodolfo Gómez Zuñiga \\
\hline Alvaro Cobo & Laura Rey \\
\hline Anxo Sánchez & Eva Loureiro \\
\hline Anxo Sánchez & José Gabriel Cataño Rojas \\
\hline Carlos Díaz & Francisco López de Ipiña \\
\hline Eva Loureiro & Lorena Fernández \\
\hline Fernando Maldonado & José Gabriel Cataño Rojas \\
\hline Gabriel Catano & Silvia Domínguez \\
\hline Gilberto Mascarenhas & Larissa Adler-Lomnitz \\
\hline Isidro Maya Jariego & Josép Vives \\
\hline Juan Brito & Miriam González \\
\hline Juan Machin Ramírez & Lorena Pasarin \\
\hline Gabriel Vélez & Laura Rey \\
\hline Laura Rey & Sergio Guerrero \\
\hline Isidro Maya Jariego & Mario Unda \\
\hline Martin Rojas & \\
\hline Nicole Verdugo & Sidro Maya Jariego \\
\hline
\end{tabular}

Al observar más de cerca la Tabla 10, es claro que hay actores mejor relacionados que otros por lo tanto tienen una posición estratégica para "pescar", en mejores condiciones, insumos de información que estén circulando al interior de la red. Esto conduce a revisar la estructura de relaciones con las categorías de centralidad.

Al analizar el grado nodal (degree) de la población en cuestión (Gráfico 5) se observan aquellos nodos que, por la cantidad de vínculos que tienen con la red social, son centrales en la recepción y envío de información (aunque también son los más susceptibles a recibir información menos sólida y/o fehaciente). También se logran distinguir nodos que no eran tan visibles en el intercambio de información sobre ARS. 
Gráfico 5. Grado nodal (degree) generado por los intercambios, vía e-mail, entre un población especializada en el ARS. 2009-2012. Programa Visone.

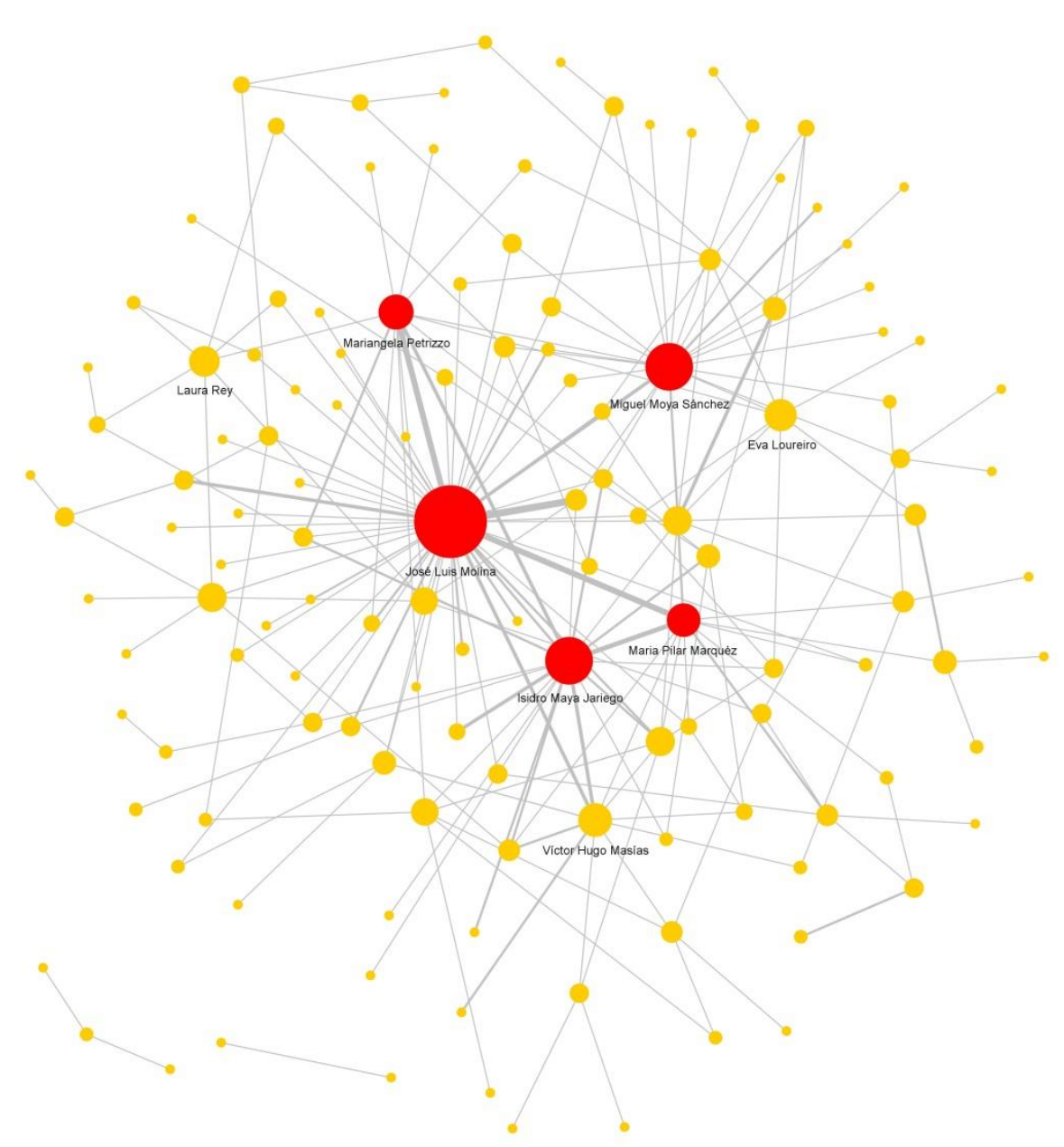

El grado de intermediación (Betwenness) señala aquellos actores que son centrales en la trama al momento de conectar a otros actores y/o grupos que no están vinculados directamente (Gráfico 6). De esta forma, surgen aquellos actores que tienen un grado mayor de legitimidad, negociación y/o contacto, vía e-mail, con los demás miembros de este colectivo, por lo tanto, son actores con un grado significativo de legitimación e influencia al interior del mismo. Sin olvidar que dicha legitimación está dada por el número, tipo y calidad de las comunicaciones que envían, reciben y/o responden con los demás adscritos. En relación con esto hay que decir que el tipo de "espacio" y/o parcela de socialización donde se lleva a cabo el proceso de intercambio e interrelación social posee, por sí mismo, límites jurídicos que no necesariamente se aplican o son los mismos a otras parcelas de la vida social. Es decir, una persona que puede ser sumamente popular en un una 
comunidad virtual, no necesariamente lo será en el espacio de la vida del mundo de lo real.

Gráfico 6. Grado de Intermediación (Betwenness) generado por los intercambios, vía e-mail, entre un población especializada en el ARS. 2009-2012. Programa Visone.

Cabe señalar la diferencia en la centralidad de los actores al comparar los resultados de la aplicación de las categorías de grado nodal y de intermediación a la misma estructura reticular. Si bien el actor central sigue manteniendo su importancia, al interior de la red, esta importancia se ve modificada de un estado relacional al otro. Algunos de los nodos que se observan como significativos, bajo la óptica del grado nodal, dejan de serlo cuando son analizados por el grado de intermediación. Esto nos habla de la precariedad del estatus social de los nodos, en el contexto del intercambio de insumos en una estructura en red: cada nodo recibe una determinada distinción social, en términos de lo que proporciona al conjunto de la estructura en red. Es decir, Somos lo que la red social obtiene de nosotros. Por otro lado, la agrupación no es solo alrededor de determinados actores sino también 
en el entorno red (Gráfico 7). De esta forma se distinguen flujos de información que corren a todo lo largo de la estructura en reticular y que señalan la presencia de flujos de información independientes de la dinámica de integración de los actores principales, es decir, nodos que establecen una circunvalación social de intercambios y flujos de información (nodos color verde) que se conectan, a su vez, con los nodos del grupo central (color rojo). Esto traería a colación la información vertida en Tabla 10.

Gráfico 7. Grado de Intermediación (Betwenness) generado por los intercambios, vía e-mail, entre un población especializada en el ARS. 2009-2012. Programa Visone.

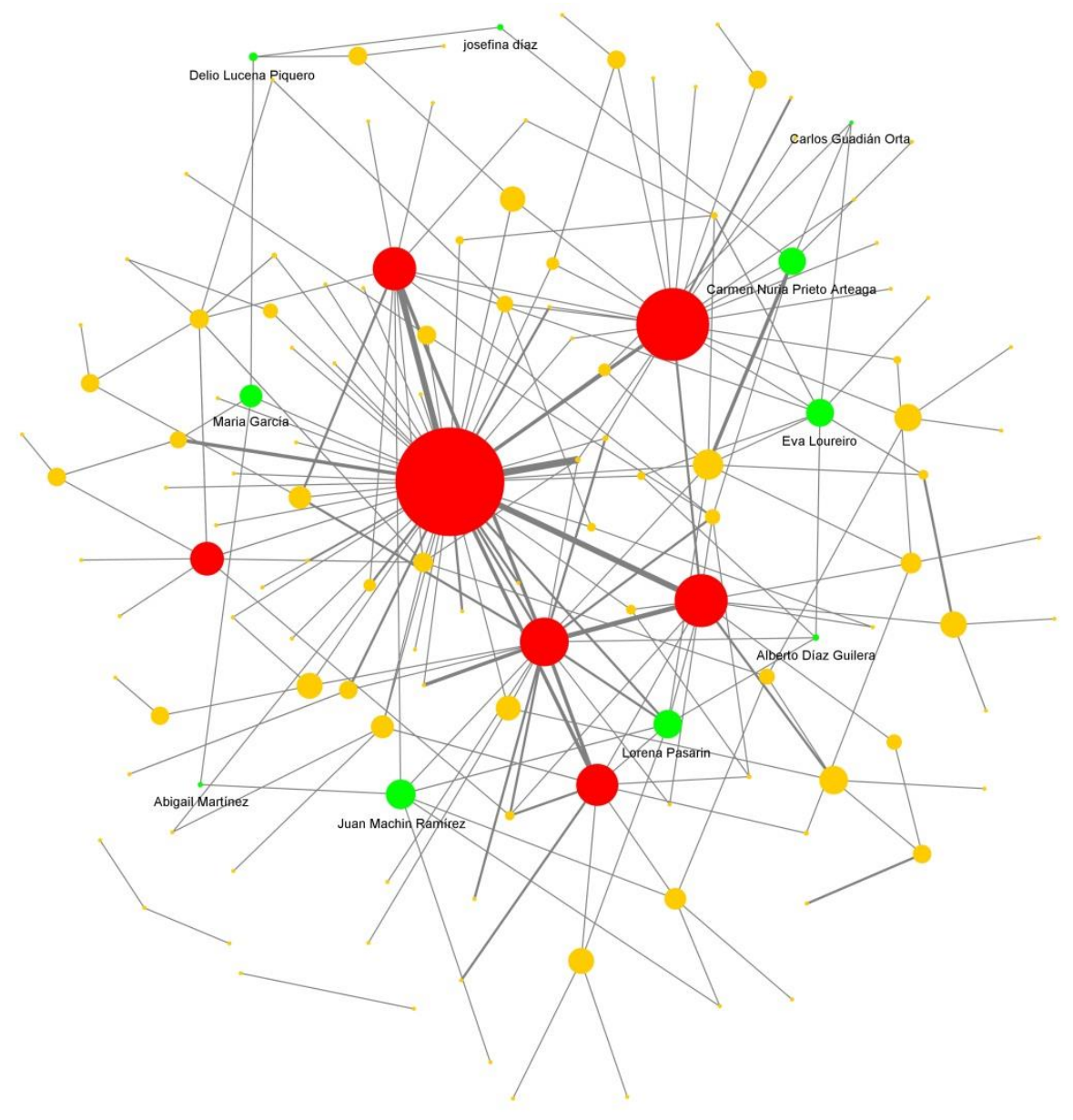

\section{Conclusiones}

Los mecanismos de agrupación de las comunidades humanas pasan por tamices y/o filtros relacionales diversos y complejos antes de poder llegar a ese punto en el cual se puede referir a un determinado grupo como comunidad. El espacio social ocupado por el desconocido pasa a ser espacio de el conocido, siempre y cuando se haya generado un proceso de contactos, intercambios y sinergias que permiten a 
los sujetos avanzar, retroceder, o seguir avanzando en la relación comunitaria. Una vez que se ha llegado a este punto, comienzan a surgir dos tipos de insumos 1) de cohesión social, tales como: confianza cooperación, solidaridad, etc. y 2) de exclusión social: límites y ordenamientos jurídicosociales (¿Qué se pude hacer, qué está prohibido hacer, con quién se puede hacer, etc.). Esto, a su vez, comienza a delinear estructuras de flujos de información y sub-grupos más o menos consolidados produciéndose núcleos relacionales y/o vecindarios sociales cercanos pero dispersos. Insumos sociales tales como saludos $\mathrm{y} / \mathrm{o}$ felicitaciones o la socialización recurrente de diversos temas (relacionados con el ARS) muestran el pilar de toda comunidad: el querer compartir.

Por otro lado, la multiplicidad de temas intercambiados en esta comunidad señala, de forma indirecta, la apertura potencial de diversos canales de comunicación, por lo tanto de socialización entre los miembros. Esto se logra observar en: a) la evolución del $\mathrm{N}^{\circ}$ de temas intercambiados por esta comunidad a lo largo de 4 años (Gráfico 1), b) en el surgimiento de grupos de intercambio al margen del integrador principal y donde surgen nodos de liderazgo "local" (Gráfico 3) y c) la apertura de canales de intercambio independientes del grupo núcleo (Gráfico 7).

A su vez, el tipo de temas que se van intercambiando en el tiempo señala la forma en la que esta comunidad va conformando su cualidad de cohesión social. En relación con esto, los 4 años revisados señalan una comunidad que se desplaza desde contactos impersonales (recomendación de web y links), hasta procesos de reconocimiento y/o renovación de contactos (saludos y felicitaciones), pasando por recomendación de bibliografía, información sobre conferencias/congresos lo que señala una mayor apertura e intimidad en el lazo social y una mayor exploración en la base epistemológica del ARS al interior de la comunidad de rederos.

Aunado a lo anterior, la construcción de una comunidad especializada en un tema determinado se expresa por el surgimiento y reiteración de elementos de cohesión/exclusión social señalados anteriormente; pero también por la especialización de dichos mecanismos. El desarrollo del ARS, en tiempos preinternet, quizás tuvo ritmos ralentizados en su dispersión y difusión (aunque con una clara evolución de su plataforma conceptual). Actualmente, su dinámica de dispersión y difusión ha adquirido ritmos vertiginosos. En relación con esto, los procesos de comunicación e intercambio de la comunidad de rederos analizada son mediados por instrumentos que poseen un alto grado de difusión, a nivel de internet, como es la recomendación de páginas, webs, links, twitter, etc. 
(podríamos decir que se genera un proceso epistovirtual). Aunque también se proponen temas de foros y medios "face to face" como conferencias, congresos e intercambio de bibliografías (esto, como medio para la exploración de la propia epistemología del ARS).

Por otro lado, la distinción de núcleos relacionales (Gráfico 4) permite observar la consolidación de una determinada retórica sobre el tema: ¿Qué autores son los más adecuados para hablar del ARS, qué escuelas son las más completas, cuales son las más cerradas y/o más abiertas, como estudiar al mismo ARS, etc.-. Este núcleo agrupa y centraliza, inevitablemente, la mayor parte de las comunicaciones. Esto, debido a que el sistema analizado es parte de su trabajo, así como a sus hábitos de uso de las tecnologías mismas. De igual forma se observan grupos periféricos. Estas agrupaciones pueden, y deben, ser temporales. Entre sus principales cualidades está el poner siempre a prueba los límites epistemológicos del tema predominante ya que permiten la introducción de nuevos flujos de información provenientes de otros grupos estudiosos del ARS, o de otras disciplinas y/o campos del conocimiento, por lo tanto permiten la expansión del propio enfoque de redes.

Es importante llamar la atención sobre la capacidad de las redes sociales para generar espacios pedagógicos y comunitarios que funcionan, por medio de la vinculación -incluso- en la dispersión geográfica. La estructura de este tipo de comunidades permite procesos pedagógicos disgregados en el mundo donde los sujetos intercambian ideas, conceptos, herramientas de análisis, etc., por lo que muchos "asisten" cotidianamente a estos espacios para aprender y explorar nuevos ángulos del propio ARS. Esta cualidad les lleva a dichos grupos ante el reto de integrarse como colectivos dispersos funcionales para fungir como think-tanks e intervenir de forma más amplia. Tal vez todo este intercambio está indicando la conformación de capitales sociales que tiene el potencial de funcionar en la dispersión: ¿Think-tanks para el análisis de problemas específicos de la glocalidad? ${ }^{3}$ ¿Aldeas glocales que hablan del ARS (por lo tanto, problemas locales estudiados con el ARS, compartidos en una comunidad diseminada en el mundo y que abonan al tema analizado)

\footnotetext{
3 Hay autores que señalan dos dimensiones de lo global (ambas interrelacionadas y simultáneas): Lo global del espacio global y lo global-local referido al establecimiento de valores globales y estructuras descentralizadas y de conectividad experimentadas a nivel local e interno de cada nación y que llaman "glocalización" (Wellman y Hampton, 2001)
} 
Si bien algunos consideran que la cualidad integradora del ARS no logra darle el cariz de paradigma, hay que tomar en cuenta que este enfoque apenas ha comenzado a ser probado en sus propios límites epistemológicos, por lo que su perfil como paradigma sólo podrá tener lugar una vez que haya sido utilizado de manera exhaustiva por la propia investigación y haya demostrado su eficacia en la explicación de lo social, global y local. En relación con esto, y desde la perspectiva de lo complejo, el ARS arroja nueva información sobre el andamiaje de la estructura social y las diferentes parcelas y/o niveles que le sustentan. Los múltiples campos de la actividad humana, que han sido observados bajo la óptica de redes sociales, señalan claramente esto.

En esta dirección el ARS permite observar, desde otro ángulo, el concepto de la aldea global (Marshall McLuhan) y su maderamen social. Este concepto es de carácter transversal y se expresa glocalmente ya que se define por una reticularidad que va desde núcleos familiares hasta asociaciones de individuos que, por sus ubicaciones geográficas, tienen poco o nulo contacto "face to face". Sin embargo, el ARS ha demostrado ser útil al momento de plantear el análisis (y la creación) de "centros" comunitarios descentralizados, aldeas glocales, que se articulan para generar procesos cotidianos de comunicación e intercambio, con consecuencias socioculturales que tienden a producir, a su vez, complejos modelos sociales interactivos que abren nuevos canales de interacción social y reconfiguración de la vida cotidiana, por lo tanto, formas organizacionales innovadas en el mundo de lo real.

Todo lo anterior lleva la intención de seguir tratando de responder preguntas que se consideran esenciales: ¿Cual es la gramática social que se va construyendo alrededor del propio ARS? ¿Se está constituyendo en un paradigma en sí y para sí (Marx) como herramienta metodológica y de intervención social? ¿Cuáles son sus límites de compresión y aprehensión de la realidad de lo social? Si es necesario responder estas preguntas, entonces analizar la construcción de las comunidades especializadas en el tema es un paso obligado en este proceso epistemológico. 


\section{Referencias bibliográficas}

Castells, Manuel (2001), La era de la información. Vol. 1, La Sociedad en Red. Alianza Ed, Madrid -1a reimpresión-.

Clark L. (2006). Manual para el mapeo de redes como una herramienta de diagnóstico. Centro Internacional de Agricultura Tropical. La Paz, Bolivia.

Gil Mendieta, Jorge; Schmidt, Samuel (2005). Estudios sobre La red política de México. Ed. UNAM-IMASS. Mexico.

Granovetter, Mark (1974). The Strength of Weak ties. American Journal of Sociology, vol. 78, n 6, 1973, pp. 1360-1380 and Getting a Job: A Study of Contacts and Careers. 1974.

Lozares C. (1996). La teoría de redes sociales. Bellaterra. pp: 103-126.

Miceli J. E. (2008). Los problemas de validez en el análisis de redes sociales: algunas reflexiones integradoras. REDES- Revista hispana para el análisis de redes sociales. Vol. 14. No. 1.

Monsalve M. M. (2008). Análisis de redes sociales: un tutorial.

Navarro S. L. A. y Salazar F. J. P. (2007). Análisis de redes sociales aplicado a redes de investigación en ciencia y tecnología. Síntesis tecnológica. Vol. 3. No. 2. pp: 69-86.

Sanz M. L. (2003). Análisis de redes sociales: o como representar las estructuras sociales subyacentes. Apuntes de ciencia y tecnología. No. 7.

Scott, John (1996). Social Network Análisis. A Handbook. Sage Publications.1996

Schmidt S. (2009). La dificultad de medir. REDES- Revista hispana para el análisis de redes sociales. Vol. 17. No. 7.

Velazquez A. O. A. y Aguilar G. N. (2005). Manual introductorio al análisis de redes sociales.

Vélez C. G. (2007). Análisis de redes sociales y teoría interorganizacional aplicados al desarrollo local regional. 1er Reunión Latinoamericana de Análisis de Redes Sociales. Universidad Nacional de la Plata. La Plata, Argentina.

The visone development team (VDT) (2012) Publicado en línea. http://visone.info

Wellman y Hampton (2001). en Anaya Dujisin, Rodrigo y Maya Jariego, Isidro. Los puentes interlocales de los universitarios alcareños en Sevilla, en Porras, José 
I; Espinoza Vicente, Redes: enfoques y aplicaciones del Análisis de Redes Sociales (ARS), Ed. Universidad Bolivariana, Chile, 2005, Pag.190.

White, Douglas; Shneegg, Michael; Brudned, Lilyan; Nutini, Hugo (2000). Conectividad múltiple, frontera e integración: Parentesco y compadrazgo en Tlaxcala rural. En Gil Mendieta, Jorge; Schmidt, Samuel. Análisis de redes: Aplicaciones en ciencias sociales. Ed. UNAM-IMASS. México. 